\title{
Finding optimum value of numerical aperture for the best aerial image quality
}

\author{
Hossein Aghababa $^{\text {a) }}$, Reza Asadpour, Ali Afzali-Kusha, \\ and Behjat Forouzandeh \\ School of ECE, University of Tehran \\ North Kargar Avenue, PO Box 14395-515, Tehran, 14399, Iran \\ a)h.aghababa@ece.ut.ac.ir
}

\begin{abstract}
Optimization of lithographic process plays a pivotal role in modern fabrication. Many parameters are engaged in optimizing optical systems used in lithographic process. In this paper, we show with a fixed rule for resolution enhancement techniques (RETs) we have an optimum value for image quality by definition of figure of merit (FOM). We find out how we are close to replicate a desired target mask on photoresist and derive an analytical formula for FOM versus numerical aperture (NA). The effect of variation in NA on quality of image that will be on photoresist is also shown.
\end{abstract}

Keywords: optimization, numerical aperture, lithography, variation Classification: Electronic instrumentation and control

\section{References}

[1] L. W. Liebmann, "Layout impact of resolution enhancement techniques: Impediment or opportunity?," Proc. Conf. International Symposium n Physical Design, Monterey, USA, pp. 110-117, April 2003.

[2] M. Yi, O. Kim, S. Bollepalli, M. Khan, and F. Cerrina, "Evolution of aerial image in XRL," Proc. Conf. Emerging Lithographic Technologies, Santa Clara, USA, pp. 126-135, March 1997.

[3] M. Fritze, B. Tyrrell, D. Astol, R. Lambert, D. Yost, A. Forte, S. Cann, and B. Wheeler, "Subwavelength optical lithography with phase-shiftmask," Lincoln Laboratory Journal, vol. 14, no. 2, pp. 237-250, 2003.

[4] J. Y. Yoo, Y. K. Kwon, J. T. Park, D. S. Sohn, I. An, H. K. Oh, and W. S. Han, "Threshold energy resist model for CD prediction," J. Appl. Phys., vol. 42, no. 6B, pp. 3905-3907, June 2003.

\section{Introduction}

Fast progress in semiconductor technology leads to fast growth in fabrication complexities. This causes an enormous need for resolution enhancement techniques (RETs) and optimization in layout and mask [1]. Many variables are effective in optical lithography like critical dimension and numerical aperture (NA). In this paper, we show that for a fixed rule of RETs there is an 
optimum value for NA to replicate a desired target mask on the photoresist. This value can be found by defining the Figure of Merit (FOM) and by this we can find a dependency between NA and FOM. For this purpose we use a figure of merit defined in [2]. The definition is based on extra and deficient energy that the photoresist receives and the desired energy of target. We derive an expression for FOM versus NA and show that it has an optimum value at which the image quality has the least sensitivity to NA variation.

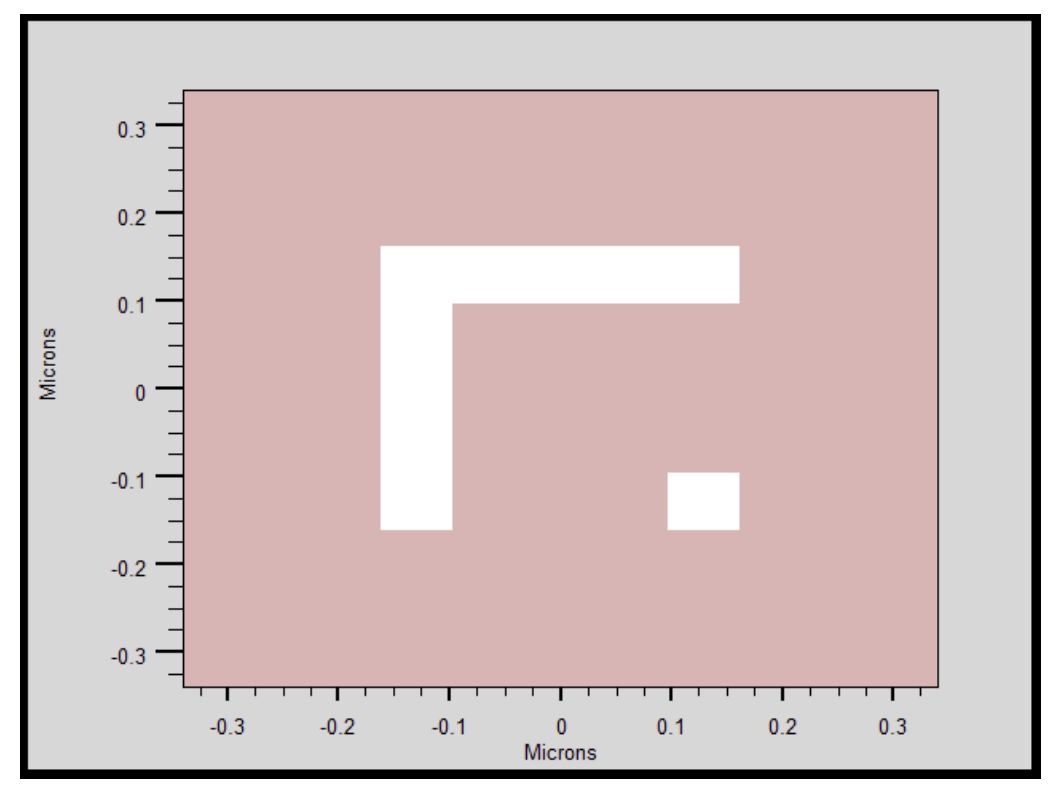

(a)

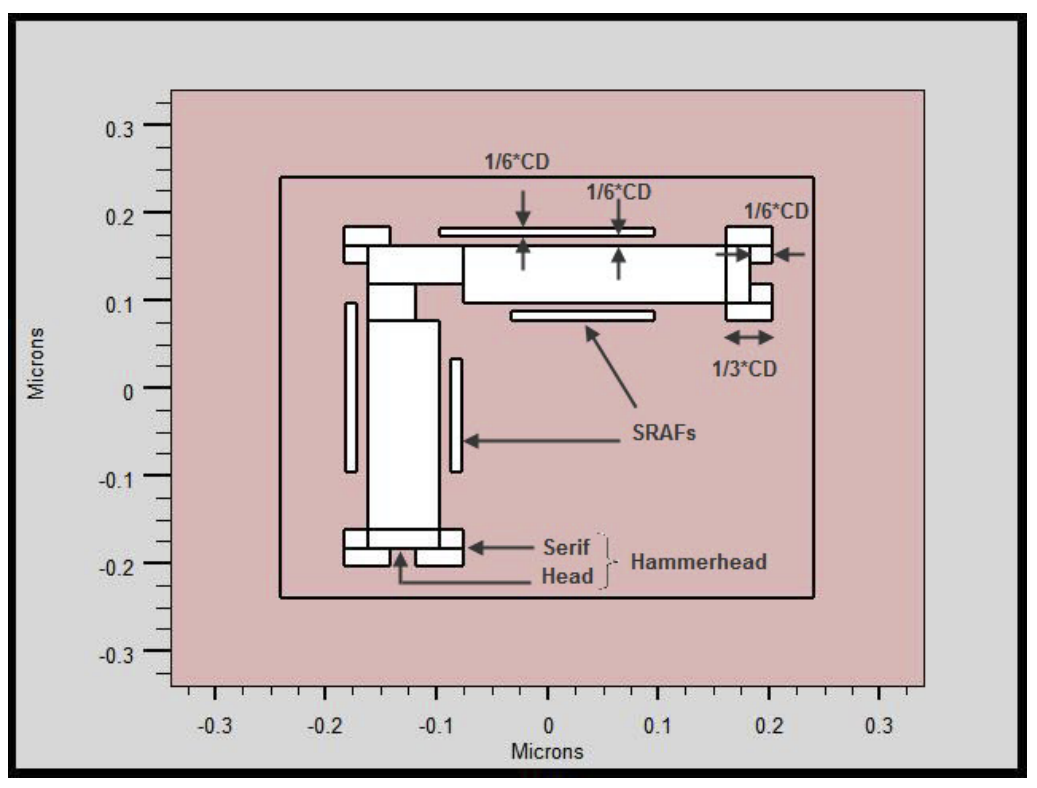

(b)

Fig. 1. (a) Shape of the mask used in simulation for both $\mathrm{CD}=65 \mathrm{~nm}$ and $45 \mathrm{~nm}$, (b) Rules of Resolution enhancement techniques for SRAFs and OPC. 


\section{Definition of FOM}

Figure of merit is a gauge to express the quality of aerial image and has a wide variety of definitions [2], but we will use the definition given in [2]. By some image processing we calculate the extra energy (EE), deficient energy (DE), and target energy (TE). By Equation (1) we have the FOM.

$$
F O M=\frac{D E+E E}{T E} .
$$

where DE is lack of energy that the target receives, EE is extra energy that the target receives, and TE is the desired energy that the target must receive. Lower FOM means better result by this definition.

\section{Simulation}

Many parameters participate in optical lithographic systems among them numerical aperture and critical dimension (CD) are considered for obtaining

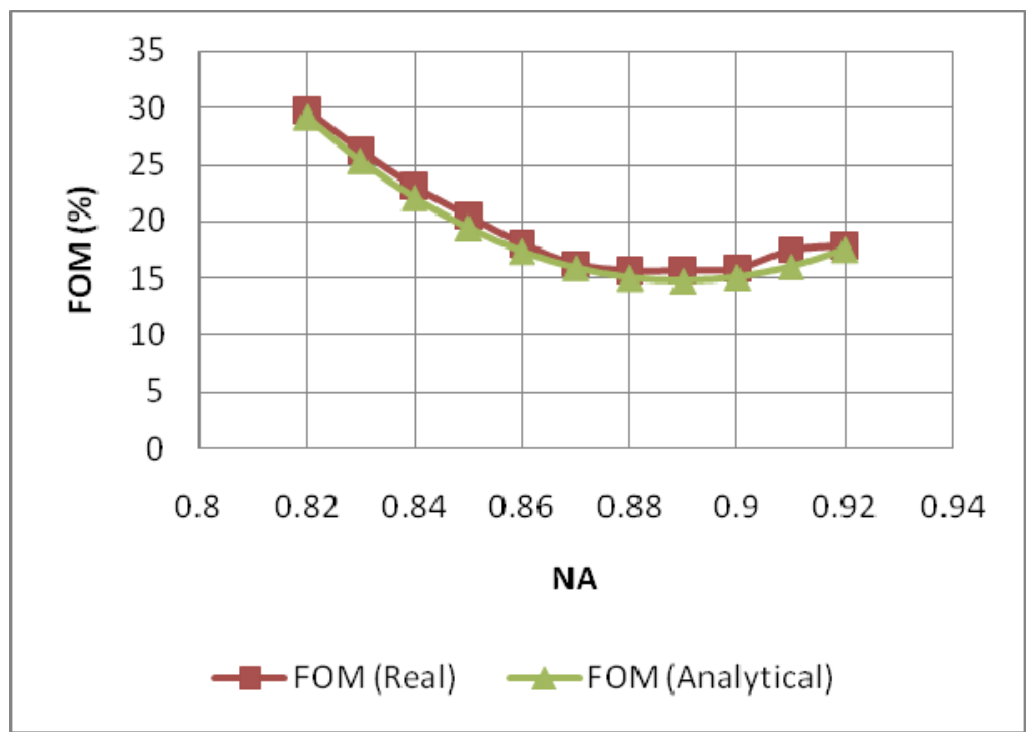

(a)

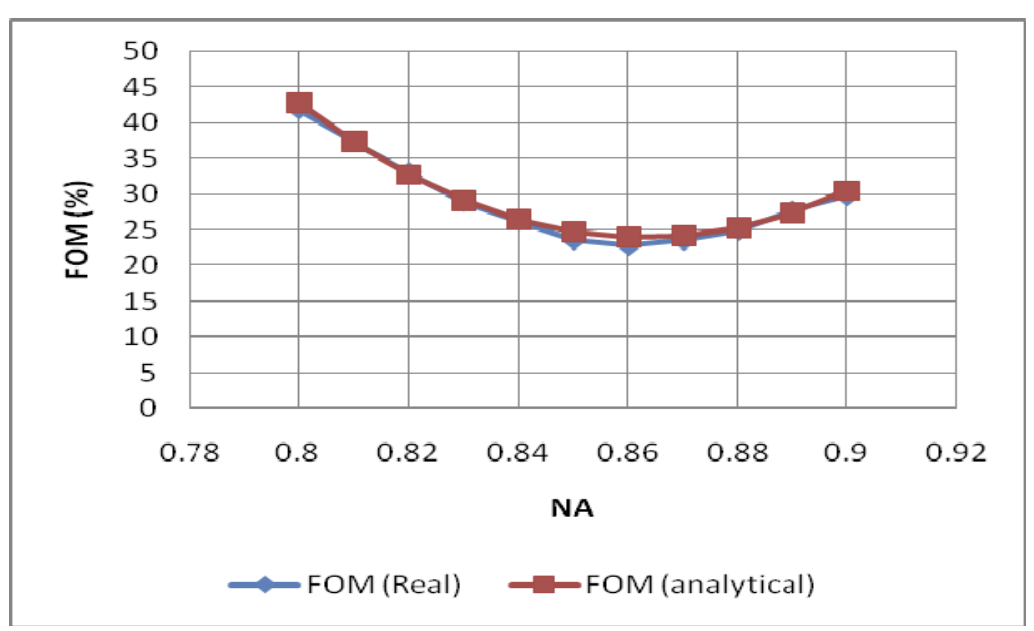

(b)

Fig. 2. (a) FOM vs. NA for $\mathrm{CD}=65 \mathrm{~nm}$, (b) FOM vs. $\mathrm{NA}$ for $\mathrm{CD}=45 \mathrm{~nm}$. 
the optimum result.

In our simulation we used an elbow and a contact hole for mask as shown in Fig. 1(a). The reason is that these shapes have many complexities that can be found in a general mask like line-end-shortening, edge rounding [3], and contact hole problems. Rules for resolution enhancement techniques are shown in Fig. 1 (b).

We used a threshold energy resist model which is one of the resist threshold models based on aerial image used for fast and simple process proximity correction [4].

The specifications of the simulation for CD $=65 \mathrm{~nm}$ and $45 \mathrm{~nm}$ are given in Table I(a).

\section{Results}

The simulation results are in Fig. 2 (a) for $\mathrm{CD}=65 \mathrm{~nm}$ and in Fig. 2 (b) for $\mathrm{CD}=45 \mathrm{~nm}$. The FOM (Analytical) results are obtained from the approximation given below (Equation (2)) while FOM (Analytical) ones are accurate results obtained from image processing.

It is obvious that for each CD we have an optimum value of FOM. By deriving the formula and finding the minimum point we will find the optimum value of NA. At this point FOM has the minimum sensitivity to variation of NA. By changing the rules of RETs we can set the optimum value of NA to desired one. The second order polynomial approximation used to fit the curve in Equation (2).

$$
F O M=a *(N A)^{2}+b *(N A)+c .
$$

Table I. (a) The specifications of the simulation for $\mathrm{CD}=$ $65 \mathrm{~nm}$ and $45 \mathrm{~nm}$, (b) Factors of FOM equation, MPE of equation, and optimum value of NA, (c) Comparison of execution time between two approaches.

(a)

\begin{tabular}{ccccccc}
\hline $\begin{array}{c}\text { shape of } \\
\text { source }\end{array}$ & $\begin{array}{c}\sigma \text { of } \\
\text { source }\end{array}$ & NA & $\begin{array}{c}\text { wave length } \\
(\lambda)\end{array}$ & $\begin{array}{c}\text { Shape of } \\
\text { pupil }\end{array}$ & CD & $\begin{array}{c}\text { Resolution } \\
\text { of } \\
\text { simulation }\end{array}$ \\
\hline Circle & 0.5 & $0.82-0.92$ & $193 \mathrm{~nm}(\mathrm{ArF})$ & Circle & $65 \mathrm{~nm}$ & $0.1 * \mathrm{CD}$ \\
Circle & 0.5 & $0.80-0.90$ & $157 \mathrm{~nm}$ & Circle & $45 \mathrm{~nm}$ & $0.1 * \mathrm{CD}$ \\
\hline
\end{tabular}

(b)

\begin{tabular}{cccccc}
\hline $\mathrm{CD}$ & $\mathrm{A}$ & $\mathrm{B}$ & $\mathrm{C}$ & $\begin{array}{c}\text { Optimum } \\
\text { NA }\end{array}$ & MPE (\%) \\
\hline $65 \mathrm{~nm}$ & 29.899 & -53.192 & 23.787 & 0.889 & 4.47 \\
$45 \mathrm{~nm}$ & 48.219 & -83.223 & 36.194 & 0.863 & 1.81 \\
\hline
\end{tabular}

(c)

\begin{tabular}{ccc}
\hline & Elapsed Time (relatively) & MPE (\%) \\
\hline Ref [2] Approach & 1 & 0 \\
Our Approach & 0.00001 & 4.47 \\
\hline
\end{tabular}


where $\mathrm{a}, \mathrm{b}$, and $\mathrm{c}$ are constant factors and NA is the numerical aperture. In Table I (b) the factors of formula, mean percent error (MPE) of the formula, and optimum value of NA are shown.

This method will improve the time needed for finding the FOM of an integrated circuit because it does not require image processing. In Table I (c) there is a time comparison between our approach and the method of [2] in the basis of their duration of execution as the unit time.

\section{Conclusion}

Many parameters affect the quality of aerial image on the photoresist which is expressed by FOM. Among them NA has a critical role. Our work shows that for a fixed set of rules of RETs the FOM versus NA has an optimum point and at this point FOM has the minimum sensitivity to NA variation. Our approach to find the FOM is faster than that of [2] especially for a complex integrated circuit and the error is still very low. In large ICs we should do more image processing which take lots of time but using a formula will decrease the execution time. 